\title{
The impact of hormone receptor on the clinical outcomes of HER2-positive breast cancer: a population-based study
}

\author{
Yiqun $\mathrm{Han}^{1} \cdot$ Yun $\mathrm{Wu}^{1} \cdot$ Hangcheng $\mathrm{Xu}^{1} \cdot$ Jiayu Wang ${ }^{1} \mathbb{D} \cdot$ Binghe $\mathrm{Xu}^{1} \mathbb{C}$
}

Received: 18 October 2021 / Accepted: 3 January 2022 / Published online: 18 January 2022

(c) The Author(s) 2022

\begin{abstract}
Background To investigate the impact of hormone receptor (HR) on the clinicopathological characteristics and prognosis of human epidermal growth factor receptor 2 (HER2)-positive breast cancer.

Methods Using the Surveillance, Epidemiology, and End Results database, we enrolled patients diagnosed with HER2positive breast cancer between 2010 and 2016, which were successively assessed for eligibility and categorized into HR + / HER2 + and HR-/HER2 + subgroups. Clinicopathological characteristics were undergone comparative analyses with the baseline distinctions calibrated by propensity score matching, while the survival outcomes were compared using KaplanMeier method with log-rank tests.

Results A total of 46,803 HER2-positive breast cancer patients were identified, of which 32,919 individuals were HR + I HER 2 + subtype and 13,884 individuals were HR-/HER2 + subtype, respectively. Comparatively, HR +/HER2 + breast cancer presented a lower histological grade, a smaller tumor size, a lower nodal involvement, and a lower rate of de novo stage IV disease. Substantial heterogeneity was detected in the metastatic patterns of organ-specific involvement between the two subgroups with initial metastasis. Overall, patients with HR +/HER $2+$ tumors had increasingly favorable prognosis in terms of overall survival and breast cancer-specific survival than patients with the HR-/HER2 + subtype. However, this kind of tendency exhibited disparities associated with HR-specific subtypes based on estrogen receptor (ER) and progesterone receptor $(\mathrm{PgR})$ status, in which $\mathrm{ER}-/ \mathrm{PgR}+$ tended to present the worst prognosis.

Conclusion This study revealed profound heterogeneity associated with HR status in the clinical outcomes of HER2-positive breast cancer regarding clinicopathological features, metastatic patterns, and prognosis. Prospective studies to optimize therapeutic strategies for HER2-positive subgroups are warranted.
\end{abstract}

Keywords Breast cancer $\cdot$ HER2-positive $\cdot$ Hormone receptor $\cdot$ Clinical features $\cdot$ Prognosis

\section{Introduction}

Breast cancer is the most common malignancy among women, with over 276,000 newly diagnosed cases and nearly 42,000 deaths annually in the United States [1]. Inherently,

Yiqun Han and Yun Wu contributed equally to this work.

Jiayu Wang

drwangjy@126.com

Binghe Xu

xubingheBM@163.com

1 Department of Medical Oncology, National Cancer Center/ National Clinical Research Center for Cancer/Cancer Hospital, Chinese Academy of Medical Sciences and Peking Union Medical College, No. 17, Panjiayuan Nanli, Chaoyang District, Beijing 100021, China breast cancer is a heterogeneous disease that is diverse in terms of molecular and clinicopathological characteristics, indicative of potentially different prognosis. According to the expression of human epidermal growth factor receptor 2 (HER2), estrogen receptor (ER), and progesterone receptor $(\mathrm{PgR})$, breast cancer can be classified into four distinctive molecular subtypes recommended as solid reference to the precise treatment and prognosis estimation [2], of which HER2 over-expression is observed in approximately 20-25\% of invasive breast cancer and is associated with an inclination of early recurrence and distant metastasis suggestive of an inferior prognosis [3].

Hormone receptors (HR), consisting of ER and PgR, are critical markers for treatment introduction of breast cancer [4]. It was acknowledged that HR-positive breast cancer, compared with HR-negative subgroup, tended to 
be decreasingly aggressive and of ameliorated prognosis due to endocrine therapy. Previous researches have managed to underline the differences in clinical characteristics, therapeutic responses, and prognosis of HER2-positive breast cancer varied by HR status [5-8]. A retrospective analysis analyzing 450 HER2-positive breast cancer patients showed that HR-negative subtype was associated with an inferior prognosis [9], while some studies indicated no significant difference existing in the long-term survival of patients between the two subgroups [10-12]. To date, given the potentially insufficient sample volume and distinct study design, the clinical impact of the HR status on the prognosis of HER2-positive breast cancer remains controversial.

In this study, based on the large-scale population, we systematically assessed the heterogeneities in the clinical outcomes, regarding clinicopathological features, metastatic patterns, and overall prognosis, of HER2-positive breast cancer associated with HR status. This knowledge was anticipated to provide an opportunity to better understand the tumor behavior and further define personalized treatment strategies for HER2-positive breast cancer patients in clinical practice.

\section{Materials and methods}

\section{Patient selection}

Data on the breast cancer patients were obtained from the Surveillance, Epidemiology, and End Results (SEER) database (2010-2016, November 2018 submission). Since HER2 status and organ-specific involvement were not registered until 2010, this study adopted the cohort dataset for which the initial diagnosis occurred after 2010. Patients were included of which the information of receptor status was complete and ruled out if the demographics and clinicopathological features were missing.

We comprehensively extracted the population demographics and clinicopathological features for each case, consisting of age at diagnosis, race, histologic type, grade, tumor size, nodal involvement, distant metastasis, HR status, HER2 status, surgical performance, radiation treatment, and chemotherapeutic delivery. Due to the publicly accessible nature of the database, this population-based analysis was exempted from approval by the ethics committee of the Chinese Academy of Medical Sciences. The current study was implemented in accordance with the Strengthening the Reporting of Observational Studies in Epidemiology (STROBE) guidelines [13] and the Transparent Reporting of a Multivariate Prediction Model for Individual Prognosis or Diagnosis (TRIPOD) statement [14].

\section{Variable definition}

In this study, HER2-positive breast cancer was classified into the HR +/HER 2 + and HR-/HER2 + subgroups with stratification by HR status. HR-specific subtypes comprised ER + / $\mathrm{PgR}+, \mathrm{ER}+/ \mathrm{PgR}-, \mathrm{ER}-/ \mathrm{PgR}+$, and $\mathrm{ER}-/ \mathrm{PgR}-$ subgroups. ER and $\operatorname{PgR}$ positivity were defined as $\geq 1 \%$ staining. Metastatic patterns were referred to the metastatic status based on the organic involvement which included bone, lung, liver, brain in accordance with the SEER terminology. Overall survival (OS) was defined as the interval from the diagnosis of breast cancer to the death caused by any reason or the last follow-up, while breast cancer-specific survival (BCSS) was the period between the initial diagnosis and cancer-related death. The American Joint Committee on Cancer $7^{\text {th }}$ edition guidelines were adopted to define the TNM staging of breast cancer.

\section{Statistical analysis}

Categorical variables, consisting of population demographics, clinicopathological characteristics, and metastatic patterns, were compared using $\chi^{2}$ tests and Fishers' exact probability tests, while continues variables were undergone comparative assessment using $t$-test for normal distribution and Mann-Whitney $U$ test for abnormally distributed variables. Survival outcomes were compared using Kaplan-Meier method with log-rank tests. Propensity score matching (PSM) was performed to further evaluate the effect of HR status on survival by eliminating objective distinctions among baseline characteristics between two subgroups of HER2-positive breast cancer. Statistical significance was set as a two-sided $P$ value less than 0.05 . For the comparison of four categories, statistical significance was determined by $P<0.05 / 6$ using Bonferroni correction to avoid multiplicity. All the statistical analyses were performed using SPSS version 26.0 (Armonk, NY, IBM Corp) and R software 3.6.4.

\section{Results}

\section{Clinicopathological characteristics}

A total of 46,803 HER2-positive breast cancer patients diagnosed from 2010 to 2016 were identified, of which 32,919 (70.3\%) were HR + /HER2 + and 13,884 (29.7\%) were HR-/HER2 + subtype. Median follow-up time for all enrolled patients was 38 months (range $0-83$ months). Duration of follow-up was longer in the HR + group (median 38 months) compared to the HR-group (median 37 months) $(P=0.011)$. The demographics and baseline characteristics 
of enrolled cohort were summarized in Appendix Table 1. The median age was 58.28 years of the HR +/HER $2+$ and was 58.11 years of the HR-/HER2 + subgroup patients $(P=0.227)$, respectively. The proportion of the white race was relatively higher in patients with $\mathrm{HR}+/ \mathrm{HER} 2+$ breast cancer $(77.1 \%$ vs. $72.3 \%, P<0.0001)$. Regarding histologic type, HR-/HER2 + tumors predisposed to be diagnosed with invasive ductal carcinoma, while the percentage of invasive lobular carcinoma was significantly higher in $\mathrm{HR}+1$ HER2 + subtype breast cancer $(P<0.0001)$. Compared to HR-/HER2 + subtype, HR +/HER2 + breast cancer had a lower tumor grade (III-IV, $51.5 \%$ vs. $74.4 \%, P<0.0001$ ), a smaller tumor size (T0-1, $49.8 \%$ vs. $43.4 \%, P<0.0001$ ), a lower nodal involvement (N0, $60.0 \%$ vs. $55.0 \%, P<0.0001$ ), and a lower rate of de novo stage IV disease (M1, 6.3\% vs. $8.0 \%, P<0.0001)$. With regard to therapeutic options, patients with HR +/HER 2 + breast cancer were more likely to receive surgery $(91.2 \%$ vs $89.4 \%, P<0.0001)$ and radiotherapy ( $45.0 \%$ vs $42.0 \%, P<0.0001)$ compared with HR-/ HER 2 + patients, while the delivery rate of chemotherapy was relatively lower in the HR +/HER 2 + subgroup patients (71.5\% vs. $76.6 \%, P<0.0001)$.

\section{Metastatic patterns}

Appendix Table 2 summarized the heterogeneity in metastatic patterns between HR +/HER 2 + and HR-/HER2 + subgroups among de novo stage IV breast cancer patients. Comparatively, HR + /HER 2 + breast cancer metastasizes in bone $(62.1 \%$ vs $43.4 \%, P<0.0001)$ at the initial diagnosis, with a declining incidence of liver (35.0\% vs $43.0 \%$, $P<0.0001)$, lung (30.2\% vs $35.4 \%, P=0.011)$, and brain involvement (6.5\% vs $9.4 \%, P=0.008)$. Concerning the single-site pattern, de novo metastatic HR +/HER2 + subtype inclined to present bone-only involvement $(30.3 \%$ vs $15.7 \%$, $P<0.0001)$ and are less manifest as lung-only $(10.1 \%$ vs $14.9 \%, P<0.0001)$ and liver-only metastasis $(10.6 \%$ vs $16.8 \%, P<0.0001)$. Furthermore, there was an increasing incidence of the paired-site pattern, comprising bone and lung $(7.3 \%$ vs $4.7 \%, P=0.004)$ as well as lung and brain $(0.2 \%$ vs $1.3 \%, P<0.0001)$, in addition to the multipleorganic pattern involving bone, liver, and brain $(0.5 \%$ vs $1.3 \%, P=0.011)$.

\section{Prognostic profiles}

An apparent discrepancy was detected in the overall prognosis of the two subgroups (Appendix Fig. 1). Patients with
HR-/HER2 + subtype breast cancer had an inferior survival, with a 3-year OS rate of $85.9 \%$ (95\% CI 85.6-86.2\%) and a 5-year OS rate of $78.8 \%$ (95\% CI 78.3-79.3\%), which was significantly decreased than that of patients from HR + /HER2 + subgroup with the a 3-year and 5-year OS rate of $90.5 \%$ (95\% CI 90.3-90.7\%) and $84.2 \%$ (95\% CI 83.9-84.5\%) $(P<0.0001)$ (Appendix Fig. 1a). This kind of distinctive profile remained consistent in the outcome regarding BCSS, which the 3-year and 5-year BCSS rate was $90.5 \%$ (95\% CI $90.2-90.8 \%$ ) and $86.1 \%$ (95\% CI $85.7-86.5 \%$ ) for HR-/HER2 + subgroup and was $94.6 \%$ (95\% CI 94.5-94.7\%) and 91.2\% (95\% CI 91.0-91.4\%) for HR +/HER $2+$ subgroup patients, respectively $(P<0.0001)$ (Appendix Fig. 1b). Regarding the HR-specific subtypes, a profound heterogeneity was demonstrated in the prognosis of HER2-positive breast cancer patients with $\mathrm{ER}+/ \mathrm{PgR}+$, $\mathrm{ER}+/ \mathrm{PgR}-, \mathrm{ER}-/ \mathrm{PgR}+, \mathrm{ER}-/ \mathrm{PgR}-$ subtypes, in which a successively worsening tendency existed in the survival with the 3-year OS rate of $91.8 \%$ (95\% CI 91.6-92.0\%), 87.8\% (95\% CI 87.4-88.2\%), 84.7\% (95\% CI 83.4-86.0\%), 85.9\% (95\% CI 85.6-86.2\%) and 5-year OS rate of $85.8 \%$ (95\% CI $85.5-86.1 \%$ ), $80.5 \%$ (95\% CI 79.9-81.1\%), $79.4 \%$ (95\% CI 77.7-81.1\%), 78.8\% (95\% CI 78.3-79.3\%) $(P<0.0001)$. For BCSS, the 3-year survival rates were $95.7 \%$ (95\% CI 95.5-95.9\%), 92.4\% (95\% CI 92.1-92.7\%), 89.9\% (95\% CI 88.8-91.0\%), 90.5\% (95\% CI 90.2-90.8\%) and 5-year survival rates were $92.6 \%$ (95\% CI 92.4-92.8\%), 88.3\% (95\% CI $87.8-88.8 \%$ ), 85.4\% (95\% CI 83.8-87.0\%), 86.1\% (95\% CI 85.7-86.5\%) of ER +/PgR +, ER +/PgR-, ER-/PgR +, ER-/PgR - groups $(P<0.0001)$, respectively (Appendix Fig. 2a, b). Results of the pairwise comparisons suggested that both the OS and BCSS of ER $+/ \mathrm{PgR}+$ subtype were significantly favorable than the others $(P<0.0001)$. No statistical significance was detected in OS and BCSS between $\mathrm{ER}+/ \mathrm{PgR}-$ and $\mathrm{ER}-/ \mathrm{PgR}+$ subgroups and in neither OS nor BCSS between ER-/PgR + and ER-/PgR- subtypes (Table S1, 2).

With the performance of PSM analysis, the heterogeneous profile of prognosis kept stable in HER2-positive breast cancer associated with HR status, which both the OS and BCSS were in favor of HR-positive subtype (3-year and 5-year OS, $87.4 \%$ vs. $86.0 \%$ and $80.0 \%$ vs. $78.9 \%, P=0.015$; 3 -year and 5-year BCSS, $92.0 \%$ vs. $90.6 \%$ and $87.3 \%$ vs. $86.1 \%$, $P=0.0017$ ) (Figure S1a, b). With regard to the prognosis associated with HR-specific subtype, the double-positive subgroup constantly exhibited the utmost favorable prognosis, while a significant divergence existed in both OS and $\mathrm{BCSS}$ between $\mathrm{ER}+/ \mathrm{PgR}+$ and $\mathrm{ER}-/ \mathrm{PgR}+$ subtype breast 
cancer (3-year and 5-year OS, $89.2 \%$ vs. $80.8 \%$ and $81.9 \%$ vs. $74.2 \%, P<0.0001$; 3-year and 5-year BCSS, $93.4 \%$ vs. $86.3 \%$ and $89.0 \%$ vs. $79.5 \%, P<0.0001$ ) (Figure S2a, b). Of note, the ER-/PgR + subtype tended to present an inferior prognosis, of which the OS $(P=0.004<0.05 / 6)$ and BCSS $(P=0.001<0.05 / 6)$ was greatly shortened than that of ER-/PgR- subtype (Table S3, 4).

Additionally, we investigated the comparative prognosis of the two subgroups of HER2-positive breast cancer associated with the organ-specific involvement (Figure S3). It was denoted that the prognosis of HR +/HER $2+$ breast cancer patients were superior to that of HR-/HER $2+$ breast cancer presenting with visceral metastasis $(P<0.0001)$, lung-only disease $(P=0.00012)$, and brain involvement $(P=0.0048)$, while no significant difference was exhibited in patients between the two subgroups with bone-only metastasis, liveronly metastasis, and brain-only metastasis.

\section{Discussion}

In this study, we curated promising impact of the HR expression on the heterogeneous outcomes of clinicopathological characteristics, metastatic patterns, and overall prognosis of HER2-positive breast cancer. To our knowledge, this was the first study that overall discussed the impact of HR status on the clinical characteristics and prognostic profiles of HER2-positive breast cancer based on a large-scale cohort, of which the findings could provide promising evidence for introduction of treatment strategies in clinical practice.

We firstly investigated the potential difference in the clinicopathological characteristics between the two subgroups of HER2-positive breast cancer. In accordance with previous data [15], the proportion of the white race was significantly advantageous in $\mathrm{HR}+$ subgroup, while the percentage of the black race was relatively higher in HR- breast cancer patients, which the individual risk factors comprising reproductive history, lactation, physical activity, mammography, and postmenopausal hormone use may explain this kind of ethnic disparity [16]. A consistent discrepancy was also detected in disease features, which HR-/HER2 + breast cancer exhibited a comparatively higher tumor grade and TNM stage, indicative of an increasing aggressiveness and progressive cancer behaviors. This proportion of results were in consistent with previous studies that reported the higher proportion of advanced stage and high-grade tumors among HR-I HER 2 + cases when compared to HR + /HER 2 + breast cancer patients $[7,11,17-20]$. Concerning treatment options, patients with HR + /HER2 + breast cancer held a climbing opportunity to receive locoregional therapeutics, such as surgery and radiotherapy, yet a less access to systemic delivery, which could be attributed to the distinctive disease factors of the two subgroups observed in the cancer course.

Notably, our investigation provided unique evidence of the associations between HR expression and metastatic patterns of newly diagnosed HER2-positive breast cancer. As expected, patients from HR + /HER 2 + subgroup presented an overwhelming frequency of bone involvement with $62.1 \%$ of the entire HER2-positive cohort, while HR-/HER2 + tumors tended to metastasize in viscera including liver and lung. This kind of strong correlation between HR status and involved organs in HER2-positive disease was proposed early in 1991 [21] and confirmed in the following studies [11, 12,22]. With an in-depth understanding of the modulated components in the various subgroups of breast cancer, there had been some studies emerging which could potentially interpret this phenomenon. For instance, the down-regulation of focal adhesion signaling in HR-negative patients was an important contributor of visceral involvement and the absence of $\mathrm{Wnt} / \beta$-catenin signaling allowed for HR-positive tumors $t$ metastasize in bone [23, 24]. In the current study, brain metastasis was more commonly occurred in the HR-negative population, which was also observed in several studies $[11,12,22,25]$ and could be the promoting profile of the conversely hyperactive $\mathrm{Wnt} / \beta$-catenin signaling pathway [23]. These results suggest that clinicians should pay more attention to the organs liable to metastasis differing by HR status for de novo stage IV breast cancer patients.

Regarding prognostic profiles, our study revealed a great heterogeneity associated with HR expression in HER2-positive breast cancer, of which the HR + expression was in line with the mortality decrease of both OS and BCSS and this kind of correlation was inherently stable. It is undeniable that adjuvant treatment including targeted and endocrine therapy could affect the prognosis of HER2-positive and HR + /HER2 + breast cancer. Yet, the proportion of patients receiving adjuvant targeted and 
endocrine therapy were unavailable in SEER database. Trastuzumab, the first humanized monoclonal antibody against HER2, has been officially approved by the FDA for the treatment of HER-2-overexpressing breast cancers in 1998. Several real-world studies [26-31] demonstrated the percentage of adjuvant target therapy based on trastuzumab-containing regimens ranged from 65.6 to $88.8 \%$ among HER 2 + breast cancer patients in the US. Previous literature has also reported that the majority of HR + patients (63.8-86\%) treated with adjuvant endocrine therapy [32-37]. Thereby, we may suppose that patients identified from SEER database have received standard adjuvant treatments. This finding was in accordance with the previous outcomes, in which the HERA trial evaluated 1703 HER 2 + early breast cancer patients underwent standard 1-year trastuzumab found a 3-year disease free survival (DFS) of $84.6 \%$ in HR + subgroup and $76.4 \%$ in HR- subgroup, respectively [38]. Likewise, a study included 3,177 patients with HER2 + breast cancer [39] showed a significantly favorable survival in patients with HR + /HER 2 + subgroup compared with HR-/HER2 + subtype with the receipt of standard adjuvant therapeutics.

A potential reason for this sort of prognostic disparity could lie in the multiple therapeutic options in the course of cancer management, especially the contents of medication therapies after recurrence. Given the considerable advances in endocrine therapy, such as fulvestrant, CDK4/6 inhibitors (ribociclib, palbociclib, abemaciclib) and everolimus, the prognosis of $\mathrm{HR}+$ metastatic breast cancer has improved immensely [40], which could lead to a superior prognosis on $\mathrm{HR}+$ patients. In addition, molecular mechanisms might partly account for the distinct prognosis of $\mathrm{HR}+1$ HER 2 + and HR-/HER2 + subtype population. Preclinical studies corroborated that PI3K, MAPK, and NOTCH were significantly overexpressed in HR-/HER 2 + breast cancer, which could be potentially correlated to therapeutic resistance and lead to the poor survival outcome [41]. Indeed, the specific influence of HR status on the overall prognosis of HER2-positive breast cancer remained controversial [42], and this kind of inconsistency may due to the relatively limited volume of sample size, insufficient follow-up, and the discordance in inclusion criteria. Under this circumstance, this large-scale, population-based study provided strong evidence for the distinctive prognosis of HR-based HER2poaistive breast cancer population. Considering the relative worse prognosis of HR-/HER2 + patients, physicians may increase the intensity of chemotherapy and targeted therapy such as the combination of trastuzumab and pertuzumab [43], and neratinib following adjuvant trastuzumab-based therapy [44] to reduce relapse rate and enhance survival in clinical practice.

As the mainstay consideration for breast cancer subtypes, the steroid hormone receptors ER and PgR are two critical biomarkers for assessing the intrinsic heterogeneity and introducing multidisciplinary therapeutics [45]. However, to date, the understanding of the clinical significance of ER and PgR status to HER2-positive breast cancer, especially regarding the HR-specific expression patterns, has been poorly investigated. Based on this cohort with extensive enrollment, we suggested that the prognosis of double-positive subtype was the most favorable in the entire population, and ER-negative exerted the foremost impact on the overall prognosis of HER2-positive breast cancer. Consistently, Bae and colleagues [46] indicated that ER + I $\mathrm{PgR}-$ and $\mathrm{ER}-/ \mathrm{PgR}+$ breast cancer were in associations with poorer DFS and OS than ER $+/ \mathrm{PgR}+$ tumors and findings of Rakha et al. [47] adopting 1,944 breast cancer patients suggested that the single-positive patterns, including $\mathrm{ER}+/ \mathrm{PgR}-$ and $\mathrm{ER}-/ \mathrm{PgR}+$, breast cancer exhibited an increasingly aggressive clinicopathological features than $\mathrm{ER}+/ \mathrm{PgR}+$ subtypes and an opposite profile in comparisons with the ER-/PgR- subtype. In this perspective, physicians could apply individual-based treatment in accordance with the varied HR-specific patterns.

The novel findings of this study lay in that the $\mathrm{ER}-/ \mathrm{PgR}+$ tumor tended to present an inferior prognosis, even to that of ER-/PgR- subtype breast cancer. This finding was in consistent with the previous study that the ER loss exerted a greater effect on the prognosis that PgR loss which could result in a poor survival of breast cancer patients [48]. In the routine practice, $\mathrm{ER}-/ \mathrm{PgR}+$ subtype breast cancer was considered as endocrine-related and would receive endocrine therapy and chemotherapy of which the protocol was with moderate strength given the estimated favorable prognosis. However, Kunc and colleagues [36] contended that patients with $\mathrm{ER}-/ \mathrm{PgR}+$ breast cancer would derive less benefit from the standard endocrine therapy than ER +1 $\mathrm{PgR}+$ disease instead of chemotherapy. In this perspective, the insufficient therapies could be attributed to the poorer survival in comparisons with the other subtypes. 
Accordingly, practitioners are supposed to use more caution to introduce reasonable therapeutics towards different HR-specific subtypes of HER2-positive breast cancer. In particular, ER-/PgR + /HER2 + subtype that is relatively insensitive to endocrine therapy, should be treated with higher-intensity chemotherapy and targeted therapy in the adjuvant setting.

Inevitably, our study has several limitations. For starters, potential selection bias introduced by the missing data could not be fully avoided due to the retrospective nature of our study. Also, therapeutic information regarding endocrine therapy and anti-HER 2 targeted therapy is not available in this database and these factors accordingly cannot be adjusted for the analysis, thereby leading to potential confounders and deviations in the study. This kind of dilemma also occurs in the absence of a few clinical parameters, such as Ki67 index, ECOG scores, and vascular invasion and part of the prognostic information in terms of recurrence-free and disease-free survival. Furthermore, although the PSM analysis were conducted to reduce the confounding factors, any bias due to the imbalance of the two groups cannot be totally excluded. Finally, the follow-up time could be insufficient by the year in which the complete information of receptor status became available in the SEER database.

\section{Conclusions}

In conclusion, this study elucidated the profound differences in clinical outcomes of HER2-positive breast cancer associated with HR status, existing across the clinicopathological features, metastatic patterns, and overall prognosis. Given the great differences in inherent behaviors and clinical outcomes of this subtype breast cancer, the HR-associated heterogeneity should be fully considered in the course of therapeutic strategies introduction and cancer management for HER2-positive breast cancer in clinical practice.

\section{Appendices}

See Tables 1, 2 and Figs. 1, 2.
Table 1 Comparative analysis of population demographics and baseline characteristics between HR +/HER 2 + and HR-/HER 2 + subgroups

\begin{tabular}{|c|c|c|c|c|c|}
\hline \multirow[t]{2}{*}{ Characteristics } & \multicolumn{2}{|c|}{$\begin{array}{l}\mathrm{HR}+/ \\
\mathrm{HER} 2+(N=32919)\end{array}$} & \multicolumn{2}{|c|}{$\begin{array}{l}\text { HR-/ } \\
\text { HER } 2+(N=13884)\end{array}$} & \multirow[t]{2}{*}{$P$ value } \\
\hline & No & Percent (\%) & No & Percent (\%) & \\
\hline $\begin{array}{l}\text { Age at diag- } \\
\text { nosis }\end{array}$ & 58.28 & & 58.11 & & 0.227 \\
\hline Race & & & & & $<0.0001$ \\
\hline White & 25397 & 77.1 & 10043 & 72.3 & \\
\hline Black & 3844 & 11.7 & 1976 & 14.2 & \\
\hline Others & 3678 & 11.2 & 1865 & 13.5 & \\
\hline Histologic type & & & & & $<0.0001$ \\
\hline Ductal & 28270 & 85.9 & 12463 & 89.8 & \\
\hline Lobular & 3588 & 10.9 & 702 & 5.1 & \\
\hline Others & 1061 & 3.2 & 719 & 5.2 & \\
\hline Grade & & & & & $<0.0001$ \\
\hline Grade1 & 2243 & 6.8 & 217 & 1.6 & \\
\hline Grade2 & 13740 & 41.7 & 3346 & 24.1 & \\
\hline Grade 3 & 16815 & 51.1 & 10202 & 73.5 & \\
\hline Grade4 & 121 & 0.4 & 119 & 0.9 & \\
\hline $\mathrm{T}$ & & & & & $<0.0001$ \\
\hline T0 & 29 & 0.1 & 18 & 0.1 & \\
\hline $\mathrm{T} 1$ & 16351 & 49.7 & 6017 & 43.3 & \\
\hline $\mathrm{T} 2$ & 12119 & 36.8 & 5152 & 37.1 & \\
\hline T3 & 2522 & 7.7 & 1368 & 9.9 & \\
\hline $\mathrm{T} 4$ & 1898 & 5.8 & 1329 & 9.6 & \\
\hline$N$ & & & & & $<0.0001$ \\
\hline No & 19740 & 60.0 & 7640 & 55.0 & \\
\hline N1mi & 1325 & 4.0 & 438 & 3.2 & \\
\hline N1 & 8105 & 24.6 & 3823 & 27.5 & \\
\hline N2 & 2285 & 6.9 & 1069 & 7.7 & \\
\hline N3 & 1464 & 4.4 & 914 & 6.6 & \\
\hline M & & & & & $<0.0001$ \\
\hline M0 & 30849 & 93.7 & 12767 & 92.0 & \\
\hline M1 & 2070 & 6.3 & 1117 & 8.0 & \\
\hline TNM & & & & & $<0.0001$ \\
\hline I & 13299 & 40.4 & 4744 & 34.2 & \\
\hline II & 12790 & 38.9 & 5364 & 38.6 & \\
\hline III & 4760 & 14.5 & 2659 & 19.2 & \\
\hline IV & 2070 & 6.3 & 1117 & 8.0 & \\
\hline Surgery & & & & & $<0.0001$ \\
\hline Yes & 30013 & 91.2 & 12415 & 89.4 & \\
\hline No/unknown & 2906 & 8.8 & 1469 & 10.6 & \\
\hline Radiotherapy & & & & & $<0.0001$ \\
\hline Yes & 14824 & 45.0 & 5830 & 42.0 & \\
\hline No/unknown & 18095 & 55.0 & 8054 & 58.0 & \\
\hline Chemotherapy & & & & & $<0.0001$ \\
\hline Yes & 23535 & 71.5 & 10634 & 76.6 & \\
\hline No/unknown & 9384 & 28.5 & 3250 & 23.4 & \\
\hline
\end{tabular}


Table2 Comparative analysis of metastatic patterns between HR + /HER 2 + and HR-/ HER2 + subgroups among de novo stage IV breast cancer patients

\begin{tabular}{|c|c|c|c|c|c|}
\hline \multirow[t]{2}{*}{ Characteristics } & \multicolumn{2}{|c|}{$\mathrm{HR}+/ \mathrm{HER} 2+(N=2070)$} & \multicolumn{2}{|c|}{ HR-/HER2 + $(N=1117)$} & \multirow[t]{2}{*}{$P$ value } \\
\hline & No & Percent (\%) & No & Percent $(\%)$ & \\
\hline \multicolumn{6}{|l|}{ Overall } \\
\hline Bone & 1286 & 62.1 & 485 & 43.4 & $<0.0001$ \\
\hline Liver & 724 & 35.0 & 480 & 43.0 & $<0.0001$ \\
\hline Lung & 625 & 30.2 & 395 & 35.4 & 0.011 \\
\hline Brain & 134 & 6.5 & 105 & 9.4 & 0.008 \\
\hline \multicolumn{6}{|l|}{ One site } \\
\hline Bone & 627 & 30.3 & 175 & 15.7 & $<0.0001$ \\
\hline Liver & 219 & 10.6 & 188 & 16.8 & $<0.0001$ \\
\hline Lung & 209 & 10.1 & 166 & 14.9 & $<0.0001$ \\
\hline Brain & 19 & 0.9 & 15 & 1.3 & 0.281 \\
\hline \multicolumn{6}{|l|}{ Two sites } \\
\hline Bone and liver & 239 & 11.5 & 110 & 9.8 & 0.154 \\
\hline Bone and lung & 151 & 7.3 & 52 & 4.7 & 0.004 \\
\hline Bone and brain & 33 & 1.6 & 13 & 1.2 & 0.356 \\
\hline Liver and lung & 60 & 2.9 & 46 & 4.1 & 0.078 \\
\hline Liver and brain & 5 & 0.2 & 5 & 0.4 & 0.334 \\
\hline Lung and brain & 5 & 0.2 & 15 & 1.3 & $<0.0001$ \\
\hline \multicolumn{6}{|l|}{ Three sites } \\
\hline Bone, liver, and lung & 130 & 6.3 & 70 & 6.3 & 0.988 \\
\hline Bone, liver, and brain & 10 & 0.5 & 15 & 1.3 & 0.011 \\
\hline Bone, lung, and brain & 24 & 1.2 & 10 & 0.9 & 0.589 \\
\hline Liver, lung, and brain & 9 & 0.4 & 5 & 0.4 & 0.958 \\
\hline \multicolumn{6}{|l|}{ Four sites } \\
\hline Bone, liver, lung, and brain & 22 & 1.1 & 19 & 1.7 & 0.139 \\
\hline
\end{tabular}

A

$+\mathrm{HR}-+\mathrm{HR}+$

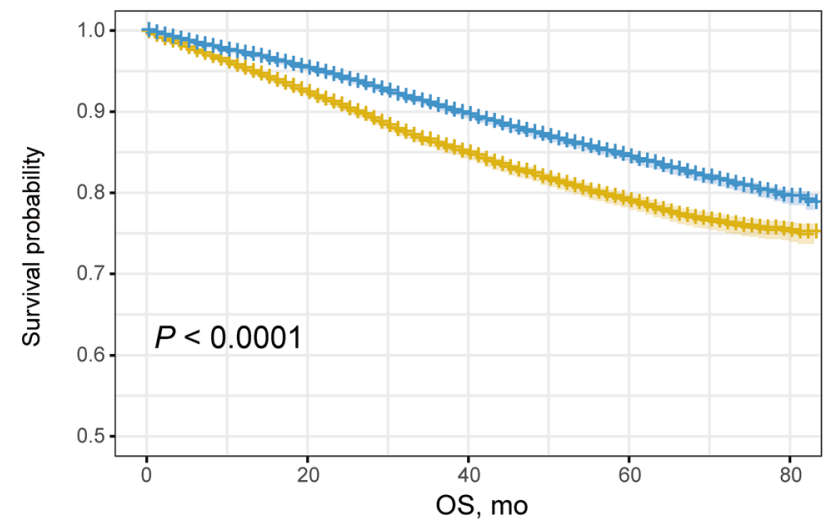

No. at risk

\begin{tabular}{|c|c|c|c|c|}
\hline 13884 & 10772 & 6426 & 3079 & 471 \\
32919 & 26170 & 15713 & 7575 & 1086 \\
\hline 1 & 20 & 40 & 60 & 80 \\
\hline
\end{tabular}

B $\quad+\mathrm{HR}-\div \mathrm{HR}+$

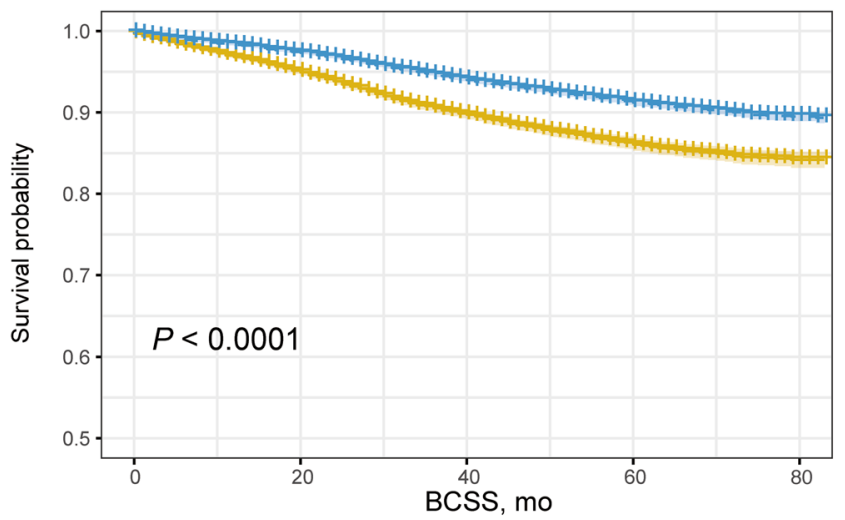

No. at risk

\begin{tabular}{|c|c|c|c|c|}
\hline 13021 & 10304 & 6221 & 3026 & 469 \\
31080 & 25035 & 15177 & 7395 & 1081 \\
\hline 1 & 20 & 40 & 60 & 80 \\
\hline 0
\end{tabular}

Fig. 1 Comparative analysis of OS (a) and BCSS (b) between HR +/HER2 + and HR-/HER2 + subgroups. HR hormone receptor, HER2 human epidermal growth factor receptor 2, OS overall survival, $B C S S$ breast cancer-specific survival 
A

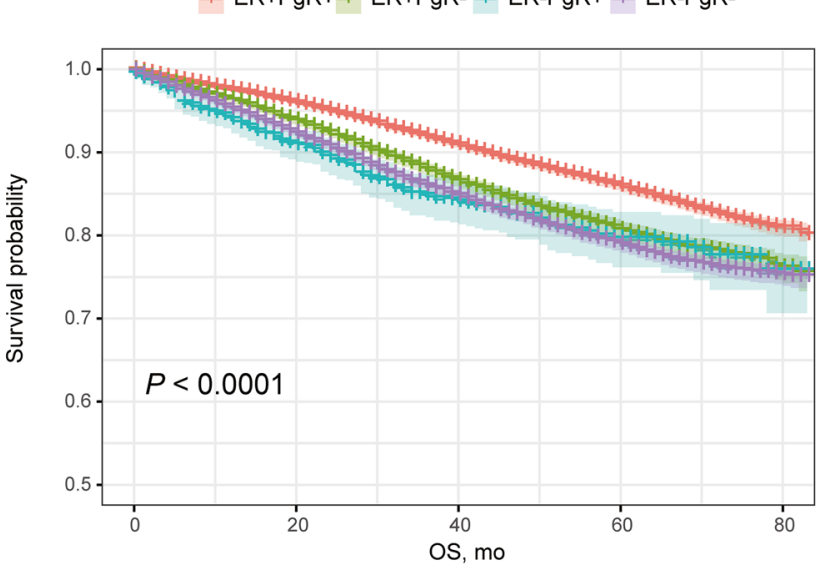

No. at risk

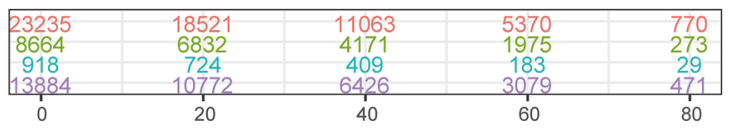

B

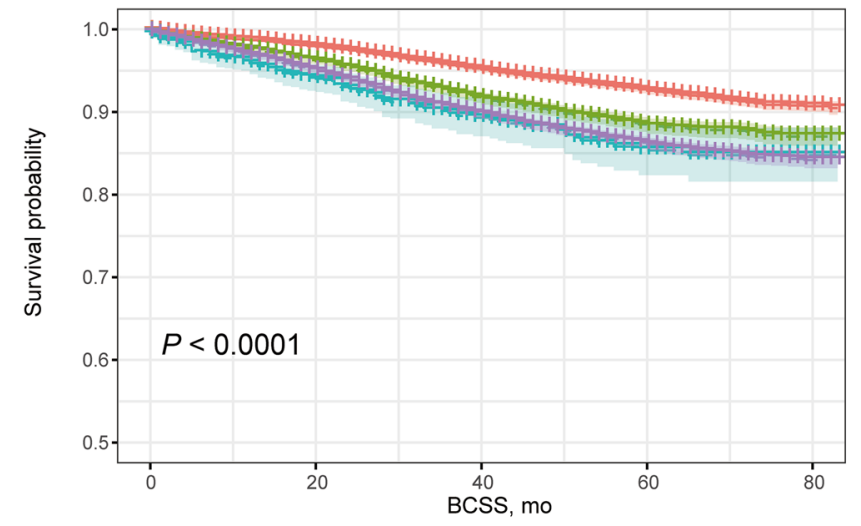

No. at risk

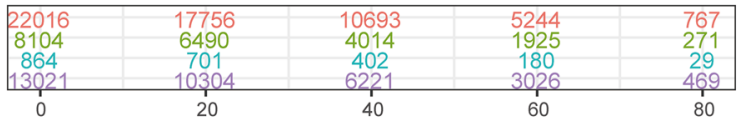

Fig. 2 Comparative analysis of OS (a) and BCSS (b) associated with ER and PgR status. ER estrogen receptor, $P g R$ progesterone receptor, $O S$ overall survival, $B C S S$ breast cancer-specific survival

Supplementary Information The online version contains supplementary material available at https://doi.org/10.1007/s10147-022-02115-x.

\section{Acknowledgements Not applicable.}

Author contribution BX and JW studied the concept and design. YH collected the data. YH and YW analyzed and interpreted the data. YW, YH and HX drafted the manuscript. JW and BX critically revised the manuscript. All authors approved the final version of the manuscript.

Funding This research was funded by China Anti-Cancer Association- Scientific Research Foundation of Anti-HER2 Targets Therapy, grant number CORP-239.

\section{Declarations}

Conflict of interest The authors declare that they have no conflict of interest.

Open Access This article is licensed under a Creative Commons Attribution 4.0 International License, which permits use, sharing, adaptation, distribution and reproduction in any medium or format, as long as you give appropriate credit to the original author(s) and the source, provide a link to the Creative Commons licence, and indicate if changes were made. The images or other third party material in this article are included in the article's Creative Commons licence, unless indicated otherwise in a credit line to the material. If material is not included in the article's Creative Commons licence and your intended use is not permitted by statutory regulation or exceeds the permitted use, you will need to obtain permission directly from the copyright holder. To view a copy of this licence, visit http://creativecommons.org/licenses/by/4.0/.

\section{References}

1. Siegel RL, Miller KD, Jemal A (2020) Cancer statistics, 2020. CA Cancer J Clin 70(1):7-30. https://doi.org/10.3322/caac.21590

2. Perou CM, Sørlie T, Eisen MB et al (2000) Molecular portraits of human breast tumours. Nature 406(6797):747-752. https://doi. org $/ 10.1038 / 35021093$

3. Balduzzi S, Mantarro S, Guarneri V et al (2014) Trastuzumabcontaining regimens for metastatic breast cancer. Cochrane Database Syst Rev. https://doi.org/10.1002/14651858.CD006242.pub2

4. Bardou VJ, Arpino G, Elledge RM et al (2003) Progesterone receptor status significantly improves outcome prediction over estrogen receptor status alone for adjuvant endocrine therapy in two large breast cancer databases. J Clin Oncol Off J Am Soc Clin Oncol 21(10):1973-1979. https://doi.org/10.1200/jco.2003.09.099

5. Blows FM, Driver KE, Schmidt MK et al (2010) Subtyping of breast cancer by immunohistochemistry to investigate a relationship between subtype and short and long term survival: a collaborative analysis of data for 10,159 cases from 12 studies. PLoS Med 7(5):e1000279. https://doi.org/10.1371/journal.pmed.1000279

6. Parise CA, Bauer KR, Brown MM et al (2009) Breast cancer subtypes as defined by the estrogen receptor (ER), progesterone receptor (PR), and the human epidermal growth factor receptor 2 (HER2) among women with invasive breast cancer in California, 1999-2004. Breast J 15(6):593-602. https://doi.org/10.1111/j. 1524-4741.2009.00822.x

7. Carey LA, Perou CM, Livasy CA et al (2006) Race, breast cancer subtypes, and survival in the Carolina Breast Cancer Study. JAMA 295(21):2492-2502. https://doi.org/10.1001/jama.295.21.2492

8. Onitilo AA, Engel JM, Greenlee RT et al (2009) Breast cancer subtypes based on ER/PR and Her2 expression: comparison of clinicopathologic features and survival. Clin Med Res 7(1-2):413. https://doi.org/10.3121/cmr.2009.825

9. Lee HJ, Park IA, Park SY et al (2014) Two histopathologically different diseases: hormone receptor-positive and hormone receptor-negative tumors in HER2-positive breast cancer. Breast 
Cancer Res Treat 145(3):615-623. https://doi.org/10.1007/ s10549-014-2983-x

10. Ribelles N, Perez-Villa L, Jerez JM et al (2013) Pattern of recurrence of early breast cancer is different according to intrinsic subtype and proliferation index. Breast Cancer Res BCR 15(5):R98. https://doi.org/10.1186/bcr3559

11. Vaz-Luis I, Ottesen RA, Hughes ME et al (2012) Impact of hormone receptor status on patterns of recurrence and clinical outcomes among patients with human epidermal growth factor2-positive breast cancer in the National Comprehensive Cancer Network: a prospective cohort study. Breast Cancer Res BCR 14(5):R129. https://doi.org/10.1186/bcr3324

12. Kennecke H, Yerushalmi R, Woods R et al (2010) Metastatic behavior of breast cancer subtypes. J Clin Oncol Off J Am Soc Clin Oncol 28(20):3271-3277. https://doi.org/10.1200/jco.2009. 25.9820

13. von Elm E, Altman D, Egger M et al (2007) The Strengthening the Reporting of Observational Studies in Epidemiology (STROBE) statement: guidelines for reporting observational studies. Lancet (London, England) 370(9596):1453-1457. https://doi.org/10. 1016/s0140-6736(07)61602-x

14. Collins G, Reitsma J, Altman D et al (2015) Transparent reporting of a multivariable prediction model for individual prognosis or diagnosis (TRIPOD): the TRIPOD statement. BMJ (Clin Res ed) 350:g7594. https://doi.org/10.1136/bmj.g7594

15. Howlader N, Altekruse SF, Li CI et al (2014) US incidence of breast cancer subtypes defined by joint hormone receptor and HER 2 status. J Natl Cancer Inst. https://doi.org/10.1093/jnci/dju055

16. Toriola AT, Colditz GA (2013) Trends in breast cancer incidence and mortality in the United States: implications for prevention. Breast Cancer Res Treat 138(3):665-673. https://doi.org/10.1007/ s10549-013-2500-7

17. Haque R, Ahmed SA, Inzhakova $G$ et al (2012) Impact of breast cancer subtypes and treatment on survival: an analysis spanning two decades. Cancer Epidemiol Biomark Prev Publ Am Assoc Cancer Res Cosponsored Am Soc Prev Oncol 21(10):1848-1855. https://doi.org/10.1158/1055-9965.Epi-12-0474

18. Park YH, Lee S, Cho EY et al (2010) Patterns of relapse and metastatic spread in HER2-overexpressing breast cancer according to estrogen receptor status. Cancer Chemother Pharmacol 66(3):507-516. https://doi.org/10.1007/s00280-009-1190-7

19. Sihto H, Lundin J, Lundin $M$ et al (2011) Breast cancer biological subtypes and protein expression predict for the preferential distant metastasis sites: a nationwide cohort study. Breast Cancer Res BCR 13(5):R87. https://doi.org/10.1186/bcr2944

20. Harvey JM, Clark GM, Osborne CK et al (1999) Estrogen receptor status by immunohistochemistry is superior to the ligand-binding assay for predicting response to adjuvant endocrine therapy in breast cancer. J Clin Oncol Off J Am Soc Clin Oncol 17(5):14741481. https://doi.org/10.1200/jco.1999.17.5.1474

21. Koenders PG, Beex LV, Langens R et al (1991) Steroid hormone receptor activity of primary human breast cancer and pattern of first metastasis. The Breast Cancer Study Group. Breast Cancer Res Treat 18(1):27-32. https://doi.org/10.1007/bf01975440

22. Gong Y, Liu YR, Ji P et al (2017) Impact of molecular subtypes on metastatic breast cancer patients: a SEER population-based study. Sci Rep 7:45411. https://doi.org/10.1038/srep45411

23. Smid M, Wang Y, Zhang Y et al (2008) Subtypes of breast cancer show preferential site of relapse. Cancer Res 68(9):3108-3114. https://doi.org/10.1158/0008-5472.Can-07-5644

24. Smid M, Wang Y, Klijn JG et al (2006) Genes associated with breast cancer metastatic to bone. J Clin Oncol Off J Am Soc Clin Oncol 24(15):2261-2267. https://doi.org/10.1200/jco.2005.03.8802

25. Brufsky AM, Mayer M, Rugo HS et al (2011) Central nervous system metastases in patients with HER2-positive metastatic breast cancer: incidence, treatment, and survival in patients from registHER. Clin Cancer Res Off J Am Assoc Cancer Res 17(14):4834-4843. https://doi.org/10.1158/1078-0432. Ccr-10-2962

26. Cossetti RJ, Tyldesley S, Speers C et al (2015) Comparison of breast cancer recurrence and outcome patterns between patients treated from 1986 to 1992 and from 2004 to 2008. J Clin Oncol Off J Am Soc Clin Oncol 33(1):65-73. https://doi.org/10.1200/ jco.2014.57.2461

27. Kurian AW, Lichtensztajn DY, Keegan TH et al (2013) Patterns and predictors of breast cancer chemotherapy use in Kaiser Permanente Northern California, 2004-2007. Breast Cancer Res Treat 137(1):247-260. https://doi.org/10.1007/s10549-012-2329-5

28. Stenehjem DD, Yoo M, Unni SK et al (2014) Assessment of HER2 testing patterns, HER2+ disease, and the utilization of HER2-directed therapy in early breast cancer. Breast Cancer (Dove Medical Press) 6:169-177. https://doi.org/10.2147/bctt. S69416

29. DaCosta Byfield S, Buck PO, Blauer-Peterson C et al (2016) ReCAP: treatment patterns and cost of care associated with initial therapy among patients diagnosed with operable early-stage human epidermal growth factor receptor 2-overexpressed breast cancer in the united states: a real-world retrospective study. J Oncol Pract 12(2):159-167. https://doi.org/10.1200/jop.2015. 004747

30. DA Patt FE, Yoo B, Wilson T et al (2016) Real-world treatment patterns and outcomes in her2 positive mbc patients with brain metastasis in the U.S. community oncology setting. J Clin Oncol Off J Am Soc Clin Oncol 34:92-92. https://doi.org/10.1200/jco. 2016.34.7_suppl.92

31. Freedman RA, Hughes ME, Ottesen RA et al (2013) Use of adjuvant trastuzumab in women with human epidermal growth factor receptor 2 (HER2)-positive breast cancer by race/ethnicity and education within the National Comprehensive Cancer Network. Cancer 119(4):839-846. https://doi.org/10.1002/cncr.27831

32. Kimmick G, Anderson R, Camacho F et al (2009) Adjuvant hormonal therapy use among insured, low-income women with breast cancer. J Clin Oncol Off J Am Soc Clin Oncol 27(21):3445-3451. https://doi.org/10.1200/jco.2008.19.2419

33. Svahn TH, Niland JC, Carlson RW et al (2009) Predictors and temporal trends of adjuvant aromatase inhibitor use in breast cancer. J Natl Compr Cancer Netw JNCCN 7(2):115-121. https://doi. org/10.6004/jnccn.2009.0011

34. Fink AK, Gurwitz J, Rakowski W et al (2004) Patient beliefs and tamoxifen discontinuance in older women with estrogen receptor-positive breast cancer. J Clin Oncol Off J Am Soc Clin Oncol 22(16):3309-3315. https://doi.org/10.1200/jco.2004.11.064

35. Cuncins-Hearn AV, Boult M, Babidge W et al (2006) National breast cancer audit: overview of invasive breast cancer management. ANZ J Surg 76(8):745-750. https://doi.org/10.1111/j.14452197.2006.03846.x

36. Ooi CW, Campbell ID, Kollias J et al (2012) National Breast Cancer Audit: overview of invasive breast cancer in New Zealand. $\mathrm{N}$ Z Med J 125(1359):7-16

37. Bowles EJ, Buist DS, Chubak J et al (2012) Endocrine therapy initiation from 2001 to 2008 varies by age at breast cancer diagnosis and tumor size. J Oncol Pract 8(2):113-120. https://doi.org/ 10.1200/jop.2011.000417

38. Untch M, Gelber RD, Jackisch C et al (2008) Estimating the magnitude of trastuzumab effects within patient subgroups in the HERA trial. Ann Oncol Off J Eur Soc Med Oncol 19(6):10901096. https://doi.org/10.1093/annonc/mdn005

39. Chumsri S, Li Z, Serie DJ et al (2019) Incidence of late relapses in patients with HER2-positive breast cancer receiving adjuvant trastuzumab: combined analysis of NCCTG N9831 (alliance) and NRG oncology/NSABP B-31. J Clin Oncol Off J Am Soc Clin Oncol 37(35):3425-3435. https://doi.org/10.1200/jco.19.00443 
40. Nagaraj G, Ma CX (2021) Clinical challenges in the management of hormone receptor-positive, human epidermal growth factor receptor 2-negative metastatic breast cancer: a literature review. Adv Ther 38(1):109-136. https://doi.org/10.1007/ s12325-020-01552-2

41. Omarini C, Bettelli S, Caprera C et al (2019) Differential molecular pathways expression in HER2 positive early breast cancer according to hormone receptor status. J Cancer Res Clin Oncol 145(4):821-828. https://doi.org/10.1007/s00432-018-02833-8

42. Cantini L, Pistelli M, Merloni F et al (2020) Body mass index and hormone receptor status influence recurrence risk in HER2-positive early breast cancer patients. Clin Breast Cancer 20(1):e89e98. https://doi.org/10.1016/j.clbc.2019.06.008

43. von Minckwitz G, Procter M, de Azambuja E et al (2017) Adjuvant pertuzumab and trastuzumab in early HER2-positive breast cancer. N Engl J Med 377(2):122-131. https://doi.org/10.1056/ NEJMoa1703643

44. Martin M, Holmes FA, Ejlertsen B et al (2017) Neratinib after trastuzumab-based adjuvant therapy in HER2-positive breast cancer (ExteNET): 5-year analysis of a randomised, double-blind, placebo-controlled, phase 3 trial. Lancet Oncol 18(12):16881700. https://doi.org/10.1016/s1470-2045(17)30717-9

45. Coates AS, Winer EP, Goldhirsch A et al (2015) Tailoring therapies-improving the management of early breast cancer: St
Gallen International Expert Consensus on the Primary Therapy of Early Breast Cancer 2015. Ann Oncol Off J Eur Soc Med Oncol 26(8):1533-1546. https://doi.org/10.1093/annonc/mdv221

46. Bae SY, Kim S, Lee JH et al (2015) Poor prognosis of single hormone receptor- positive breast cancer: similar outcome as triplenegative breast cancer. BMC Cancer 15:138. https://doi.org/10. 1186/s12885-015-1121-4

47. Rakha EA, El-Sayed ME, Green AR et al (2007) Biologic and clinical characteristics of breast cancer with single hormone receptor positive phenotype. J Clin Oncol Off J Am Soc Clin Oncol 25(30):4772-4778. https://doi.org/10.1200/jco.2007.12. 2747

48. Li Y, Yang D, Yin X et al (2020) Clinicopathological characteristics and breast cancer-specific survival of patients with single hormone receptor-positive breast cancer. JAMA Netw Open 3(1):e1918160. https://doi.org/10.1001/jamanetworkopen.2019. 18160

Publisher's Note Springer Nature remains neutral with regard to jurisdictional claims in published maps and institutional affiliations. 